\title{
Interplay among unstable modes in films over permeable walls
}

\author{
C. Camporeale ${ }^{1} \dagger$, E. Mantelli ${ }^{1}$ and C. Manes ${ }^{2}$ \\ ${ }^{1}$ Department of Environmental, Land and Infrastructure Engineering, Politecnico di Torino, Corso Duca \\ degli Abruzzi 24, 10129 Torino, Italy \\ ${ }^{2}$ Faculty of Engineering and the Environment, University of Southampton, Highfield, Southampton SO17 \\ $1 \mathrm{BJ}, \mathrm{UK}$
}

(Received 23 August 2012; revised 11 December 2012; accepted 2 January 2013)

The stability of open-channel flows (or film flows) has been extensively investigated for the case of impermeable smooth walls. In contrast, despite its relevance in many geophysical and industrial flows, the case that considers a permeable rather than an impermeable wall is almost unexplored. In the present work, a linear stability analysis of a film falling over a permeable and inclined wall is developed and discussed. The focus is on the mutual interaction between three modes of instability, namely, the well-known free-surface and hydrodynamic (i.e. shear) modes, which are commonly observed in open-channel flows over impermeable walls, plus a new one associated with the flow within the permeable wall (i.e. the porous mode). The flow in this porous region is modelled by the volume-averaged Navier-Stokes equations and, at the wall interface, the surface and subsurface flow are coupled through a stress-jump condition, which allows one to obtain a continuous velocity profile throughout the whole flow domain. The generalized eigenvalue problem is then solved via a novel spectral Galerkin method, and the whole spectrum of eigenvalues is presented and physically interpreted. The results show that, in order to perform an analysis with a full coupling between surface and subsurface flow, the convective terms in the volume-averaged equations have to be retained. In previous studies, this aspect has never been considered. For each kind of instability, the critical Reynolds number $\left(R e_{c}\right)$ is reported for a wide range of bed slopes $(\theta)$ and permeabilities $(\sigma)$. The results show that the free-surface mode follows the behaviour that was theoretically predicted by Benjamin and Yih for impermeable walls and is independent of wall permeability. In contrast, the shear mode shows a high dependence on $\sigma$ : at $\sigma=0$ the behaviour of $\operatorname{Re}_{c}(\theta)$ recovers the well-known non-monotonic behaviour of the impermeable-wall case, with a minimum at $\theta \sim 0.05^{\circ}$. However, with an increase in wall permeability, $R e_{c}$ gradually decreases and eventually recovers a monotonic decreasing behaviour. At high values of $\sigma$, the porous mode of instability also occurs. A physical interpretation of the results is presented on the basis of the interplay between the free-surfaceinduced perturbation of pressure, the increment of straining due to shear with the increase in slope, and the shear stress condition at the free surface. Finally, the 
paper investigates the extent to which Squire's theorem is applicable to the problem presented herein.

Key words: interfacial flows (free surface), porous media, shear waves

\section{Introduction}

The stability analysis of film flows over an inclined plane is a subject that has been extensively studied. With respect to closed-conduit flows, the hydrodynamic stability of films is driven, besides the Reynolds number $(R e)$, by the slope $\theta$ of the plane as a free parameter. The seminal papers by Benjamin (1957), Yih (1963), Debruin (1974) and Floryan, Davis \& Kelly (1987) elucidated that film flows over smooth surfaces experience two types of instability, namely a surface mode and a shear mode. The former is associated with long surface waves propagating at twice the mean fluid speed, and the latter with essentially Tollmien-Schlichting waves that propagate at velocities lower than the mean fluid speed. Benjamin (1957) and Yih (1963) have shown that the critical Reynolds number $R e_{c}$ for the surface mode varies as $\sim \cot \theta$. Debruin (1974) and Floryan et al. (1987) pointed out that the critical Reynolds number for the shear mode varies non-monotonically with the inclination angle, displaying a minimum at $\theta=3.214^{\prime} \sim 0.05^{\circ}$. Furthermore, they observed that the critical $R e$ for the shear mode is always higher than that of the surface mode except for very small inclination angles, with the cross-over occurring at $\theta=1^{\prime}$. Further studies have presented linear stability analysis of film flows by progressively increasing the complexity of the problem, e.g. by adding bottom heating (Trevelyan et al. 2007; Thiele, Goyeau \& Velarde 2009), undulated bottom topography (Myers 2003), movable bottom topography (Devauchelle et al. 2010; Camporeale \& Ridolfi 2012a), permeable substrates (Pascal 1999; Sadiq \& Usha 2008) or combinations of these (D’Alessio et al. 2010; Ogden, D'Alessio \& Pascal 2011).

The subject of this paper is the stability analysis of isothermal flows over permeable substrates, which is a problem that has been less explored than the impermeable case and requires further analysis and clarification. The relevance of this study lies in the fact that many geophysical and industrial flows, such as open-channel flows over granular beds (e.g. sand or gravel), film flows over melting snow (Hutter 1983) and paper coating flows (Aidun 1991), occur over permeable substrates. Understanding whether such flows occur in laminar or turbulent conditions is a fundamental prerequisite for the modelling of relevant processes (e.g. mass and heat transfer or coating efficiency) and, for this purpose, linear stability analysis represents a valuable research tool.

The problem of isothermal flows over permeable substrates was first dealt with by Pascal (1999), who performed a linear stability analysis of a one-sided model, where the effects of the permeable substrate were reduced to a boundary condition at the fluid-wall interface, hence neglecting the coupling between the subsurface and the surface flow. Liu \& Liu (2009) made a step forwards and performed a two-sided (i.e. coupled) analysis, although the subsurface flow was simply modelled by means of Darcy's law, and the presence of the so-called Brinkman layer was neglected. This layer identifies a flow region located in the proximity of the wall interface and it is characterized by a gradient in the mean velocity profile caused by the shear imposed by the overlying surface flow. At the fluid-wall interface, they employed the well- 
known interface condition proposed by Beavers \& Joseph (1967). The results of Liu \& Liu (2009) showed that the coupled and uncoupled models provide very similar results. Furthermore, they identified a third unstable mode associated with the flow within the porous medium. The stability of all three of these modes was then investigated as a function of the plane inclination, the depth ratio (i.e. the ratio between flow depth and thickness of the porous medium), the Darcy number (i.e. the ratio between the square root of permeability and the thickness of the porous medium) and the empirical constant $\alpha_{B J}$ contained within the Beavers and Joseph interface condition, which essentially defines the magnitude of the mean velocity gradient at the interface. The main results from this analysis showed that the stability of the surface mode depends on both the depth ratio and the Darcy number and only weakly on $\alpha_{B J}$.

Within the context of stability analysis of shear flows over permeable walls, the work by Tilton \& Cortelezzi (2008) on closed-channel flows (i.e. without a free surface) is the one that, in the authors' opinion, provides the most rigorous analysis. Tilton \& Cortelezzi (2008) pointed out that the range of free parameters that can be investigated must be consistent with the equations of motion and the boundary conditions associated with the problem. In particular, they were particularly careful in investigating a range of free parameters (e.g. the normalized wall permeability) for which the assumption of negligible inertial effects in the equation of motion describing the flow within the permeable region was satisfied. Such a condition facilitates the stability analysis enormously, since it allows one to neglect terms that are either nonlinear, and hence incompatible with the concept of linear stability analysis, or very difficult to parametrize. As a rule of thumb, Tilton \& Cortelezzi (2008) suggested that inertial effects can be considered negligible if the ratio between the square root of permeability and the channel half-width does not exceed 0.02. In the literature pertaining to film flows, the assumption of negligible inertial effects has been commonly employed, but the range of permeabilities investigated often far exceeds the limit suggested by Tilton \& Cortelezzi (2008). For example, Liu \& Liu (2009) extend their analysis to values that exceed this limit by factors of 2 and 3, and therefore it is difficult to establish the relevance of their results.

This paper extends the approach of Tilton \& Cortelezzi (2008) to the case of open-channel flows, with a focus on the physics underlying the different types of instabilities and their mutual interactions. In the first part of the paper, we derive the equations of motion inside and outside the porous wall and assess the terms within the equations that should be retained in order to perform a two-sided linear stability analysis. We pay particular attention to the role of the convective terms appearing in the equation of motion of the subsurface flow. We show that neglecting these terms implies decoupling the basic state in the Brinkman layer from the whole stability analysis, with the paradoxical consequence that the instabilities are independent of the shape of the basic state. This aspect has passed unnoticed in the literature so far. The linear stability analysis is then carried out for an extensive set of inclination angles and wall permeabilities in order to investigate their effects on the surface, shear and porous instability. To this aim, a novel spectral solution has allowed the whole eigenspectrum to be obtained with high accuracy. A complex and varied scenario of instabilities is then discussed by paying particular attention to the interactions among the three kinds of instability. A physical interpretation of the results is presented on the basis of the interplay between the free-surface-induced perturbation of pressure, the increment of straining due to shear with the slope, and the shear stress condition at the free surface. This has also permitted us to explain the reasons for the sag-like curve, which was observed some time ago by Debruin (1974) and Floryan et al. (1987) without any 
(a)

(b)
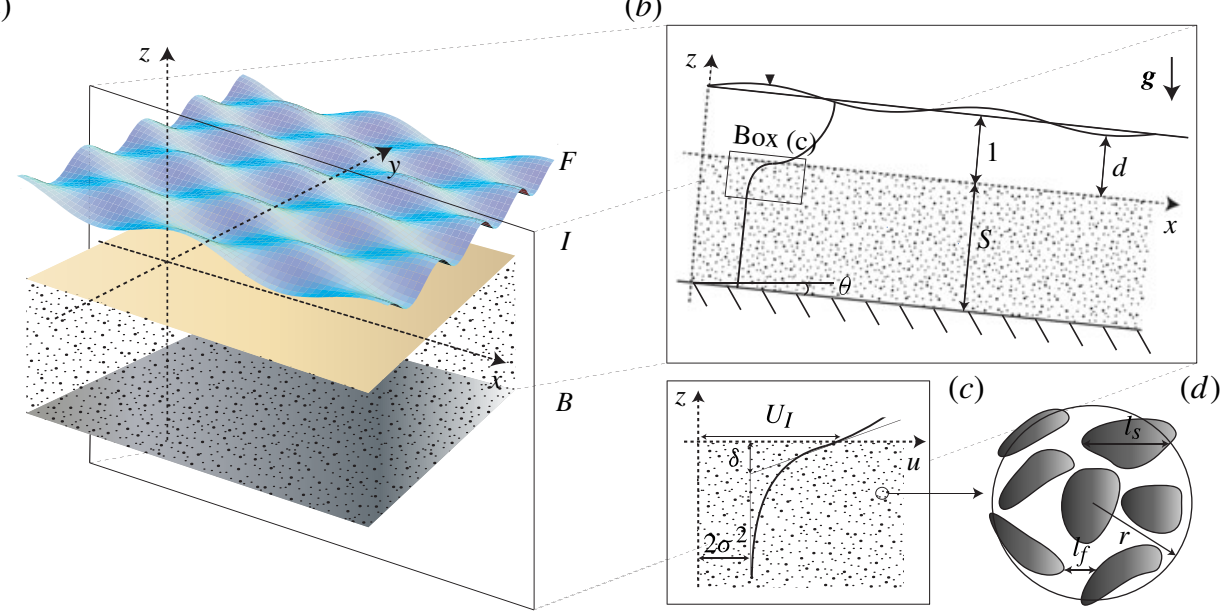

FIGURE 1. (Colour online) Sketch of the physical problem with dimensionless quantities.

justification. In the last part of the paper, we discuss how three-dimensional effects influence shear and porous instabilities, showing that Squire's theorem is not entirely applicable to the problem presented herein.

\section{Mathematical problem}

\subsection{Governing equations}

The analysis is carried out for an incompressible viscous film flowing on a flat porous wall that is inclined at an angle $\theta$ (see figure 1). The right-handed Cartesian reference frame, $\tilde{\boldsymbol{x}} \equiv\{\tilde{x}, \tilde{y}, \tilde{z}\}$, is employed here, where $\tilde{x}$ is tangent to the base plane (spanned by the vector $\left.\tilde{\boldsymbol{x}}_{h} \equiv\{\tilde{x}, \tilde{y}\}\right)$ and parallel to the direction of the maximum slope, and $\tilde{z}$ is orthogonal to the base plane and points upwards. Accordingly, $\tilde{u}, \tilde{v}$ and $\tilde{w}$ are the components of the velocity vector $\boldsymbol{u}(\tilde{\boldsymbol{x}}, \tilde{t})$ and $\tilde{p}$ is pressure. The upper boundary of the domain (i.e. the free surface) is defined by $\mathscr{F}=\tilde{z}-\tilde{h}\left(\tilde{\boldsymbol{x}}_{h}, t\right)=0$, while the lower boundary is delimited by an impermeable wall located at $\tilde{z}=-\tilde{S}$. Henceforth, the tilde refers to the dimensional variables and the subscripts $F, I$ and $B$ refer to the free surface, the liquid-porous medium interface, and the bottom of the solid phase, respectively.

The porous region is assumed to be a two-phase system characterized by solid $\left(\tilde{l}_{s}\right)$ and fluid $\left(\tilde{l}_{f}\right)$ length scales that are much smaller than the macroscopic length scale of the porous medium, $\tilde{L}=O(\tilde{S})$. According to Whitaker (1986), the method of volume averaging allows one to capture the large-scale behaviour of the flow in the porous medium, by averaging the momentum and continuity equations over a volume, $\tilde{V}$, of radius $\tilde{r}$, provided that $\tilde{l}_{s} \sim \tilde{l}_{f} \ll \tilde{r} \ll \tilde{L}$ (figure $1 d$ ). In this way, the superficial volume average, $\langle a\rangle=\tilde{V}^{-1} \int_{\tilde{V}} a \mathrm{~d} \tilde{V}$ of any quantity, $a$, is associated with the centroid of the volume, $\tilde{V}$, and the porous medium is treated as a continuum.

In order to consider a suitable non-dimensionalization of the problem, we refer to the unperturbed case of a film flowing over an impermeable wall. This is the well-known Nusselt problem, for which the following solutions for the depth of the 
film, $\tilde{D}$, and the free-surface velocity, $\tilde{U}_{F}$, hold:

$$
\tilde{D}=\left(\frac{2 v^{2} R e}{g \sin \theta}\right)^{1 / 3}, \quad \tilde{U}_{F}=\left(\frac{g v}{2} R e^{2} \sin \theta\right)^{1 / 3},
$$

where $v$ is the kinematic viscosity, $g$ is the gravity acceleration and $\operatorname{Re}=\tilde{U}_{F} \tilde{D} / v$ is the bulk Reynolds number of the film. Hence, normalization is carried out as follows: given the slope $\theta$ and the depth $\tilde{D}$ of a film flowing over a porous medium characterized by a porosity $\epsilon$ and a permeability $\tilde{k}$, the variables will be made dimensionless using $\tilde{U}_{F}$ and $\tilde{D}$, corresponding to the non-porous case via $(2.1)$, and having the same depth and slope.

With the above formalism, the dimensionless Navier-Stokes equations in the film and porous domains read (Whitaker 1986)

$$
\begin{aligned}
& {\left[\frac{\partial}{\partial t}+\boldsymbol{u}_{l} \cdot \nabla-\frac{\nabla^{2}}{R e}\right] \boldsymbol{u}_{l}+\nabla p_{l}+g=0, \quad \nabla \cdot \boldsymbol{u}_{l}=0 \quad\left(0<z<h\left(\boldsymbol{x}_{h}, t\right)\right),} \\
& {\left[\frac{\partial}{\partial t}+\frac{\left\langle\boldsymbol{u}_{p}\right\rangle \cdot \nabla}{\epsilon}-\frac{\nabla^{2}}{\operatorname{Re}}+\frac{\epsilon}{\sigma^{2} \operatorname{Re}}\right]\left\langle\boldsymbol{u}_{p}\right\rangle+\nabla\left\langle p_{p}\right\rangle+\epsilon \boldsymbol{g}=0, \quad \nabla \cdot\left\langle\boldsymbol{u}_{p}\right\rangle=0 \quad(-S<z<0) .}
\end{aligned}
$$

Here the subscripts $l$ and $p$ refer to the liquid and porous regions, respectively; $\boldsymbol{g}=\Delta\{-\tan \theta, 0,1\} ; \Delta=\cos \theta / F r^{2} ; F r=\tilde{u}_{F}(g \tilde{D})^{-1 / 2}$ is the Froude number; and $\sigma=\sqrt{\tilde{k}} / \tilde{D}$ is the dimensionless permeability of the porous medium, which is here assumed to be thicker than the Brinkman layer, i.e. $S \gg \delta$. The square brackets in the above equations contain (from left to right) the unsteady, convective and diffusion terms plus, in (2.3a) only, the drag term parametrized with the Darcy's law. Finally, $\boldsymbol{g}$ accounts for gravity, which in open-channel flows represents the main momentum source.

It should be noted that, in the derivation of (2.3), two terms have been neglected: (i) the so-called volume filter, $\nabla \cdot\left\langle\hat{\boldsymbol{u}}_{p} \cdot \hat{\boldsymbol{u}}_{p}\right\rangle$ (where $\hat{\boldsymbol{u}}_{p}=\boldsymbol{u}_{p}-\left\langle\boldsymbol{u}_{p}\right\rangle$ ), which is a result of the volume averaging of the convective term $\langle\boldsymbol{u} \cdot \nabla \boldsymbol{u}\rangle$ ); and (ii) the Forchheimer correction to the drag term, which is associated with pressure forces acting around solid elements. Following Whitaker (1996), the latter correction can be neglected since it is $O\left(v_{p}^{2}\right), v_{p}$ being a characteristic value of $\left\langle\boldsymbol{u}_{p}\right\rangle$. Dealing with the volume filter is more problematic. To the best of the authors' knowledge there are no accepted theories that provide a parametrization for this term because it is associated with the covariance of the spatial velocity fluctuations and hence to the specific geometry of the solid matrix composing the porous medium. It is likely, however, that neglecting this term is a weak hypothesis for sponge-like media (e.g. foams, see Manes, Poggi \& Ridolfi 2011), which are characterized by voids that are much larger than the characteristic size of the solid elements (i.e. $\tilde{l}_{s}>\tilde{l}_{f}$ ), whereas it may represent a stronger hypothesis for granular media, where these two length scales are comparable in size.

Following a rationale similar to the one introduced for the Forchheimer correction, the convective term appearing in (2.3), has commonly been neglected in the previous literature on the basis that the characteristic inertial length scale is larger than $S$. However, in the present problem, a further inertial scale, $\delta<S$, has to be considered. This scale is related to the vertical profile of the unperturbed flow velocity in the porous medium and accounts for the depth of the region that is kinematically affected by the interface, known as the Brinkman layer (see figure $1 c$ ). With the use of $\delta$ and 
the interface velocity $U_{I}$, the criterion for neglecting the convective term in the porous region becomes $\operatorname{Re} \ll \delta /\left(\sigma^{2} U_{I}\right):=K_{c}$. It will be shown that $K_{c}$ is a function of the parameters $\sigma, \epsilon$ and $\tau$, and that this condition is not satisfied in a wide region of parameter space. Moreover, it will be revealed that the convective terms play a key role in the stability of the flow within the porous region.

The following boundary conditions complete the mathematical problem:

$$
\begin{gathered}
\frac{\mathrm{D} \mathscr{F}}{\mathrm{D} t}=0, \quad(\boldsymbol{n} \cdot \boldsymbol{T} \cdot \boldsymbol{t})_{F}=0, \quad(\boldsymbol{n} \cdot \boldsymbol{T} \cdot \boldsymbol{n})_{F}+\frac{R e}{W e} \mathscr{K}_{F}=0, \\
\boldsymbol{u}_{I}=\langle\boldsymbol{u}\rangle_{I}, \quad(\boldsymbol{n} \cdot \boldsymbol{T} \cdot \boldsymbol{n})_{I}=(\boldsymbol{n} \cdot\langle\boldsymbol{T}\rangle \cdot \boldsymbol{n})_{I}, \\
\frac{\sigma}{\epsilon} \frac{\partial}{\partial z}\left[\langle u\rangle_{I}-\epsilon u_{I}\right]=\tau u_{I}, \quad \frac{\sigma}{\epsilon} \frac{\partial}{\partial z}\left[\langle w\rangle_{I}-\epsilon w_{I}\right]=\tau w_{I}, \\
\left(\boldsymbol{u}_{p} \cdot \boldsymbol{n}\right)_{B}=0, \quad(\boldsymbol{n} \cdot\langle\boldsymbol{T}\rangle \cdot \boldsymbol{t})_{B}=0 .
\end{gathered}
$$

Here $\boldsymbol{n}$ and $\boldsymbol{t}$ are the unit normal and tangent vectors to a generic surface, respectively; $\boldsymbol{T}=p \operatorname{Re} \boldsymbol{I}-2 \boldsymbol{D}$ is the dimensionless Newtonian stress tensor (with $\boldsymbol{I}$ and $\boldsymbol{D}$ the identity matrix and the rate-of-strain tensor, respectively); $\mathscr{K}_{F}=\left[\left(\boldsymbol{I}-\boldsymbol{n}_{F}: \boldsymbol{n}_{F}\right) \cdot \nabla\right] \cdot \boldsymbol{n}_{F} / 2$ is the local mean curvature of the free surface. Moreover, $W e=(\operatorname{Re} \sin \theta) / 2 l_{c}^{2}$ is the Weber number, whereby the (dimensionless) capillary length $l_{c}$ accounts for capillary effects, whereas $\tau$ is a coefficient varying roughly between -1 and 1.5 (the common choice $\tau=0$ refers to the so-called Brinkman condition).

Equation $(2.4 a)$ is the kinematic condition that forces the free surface to be the upper boundary of the fluid domain. Equations $(2.4 b, c)$ define the so-called dynamic conditions that are associated with the conservation of momentum along the tangential and normal components of the free surface, respectively. Equations (2.5) refer to the continuity of velocity and normal stress at the interface between the liquid and porous regions. Notice that $(2.5 b)$ is more rigorous than simply setting the continuity of pressure because of the contribution of $\boldsymbol{D}$ to the normal stress, a detail that is often disregarded (e.g. Tilton \& Cortelezzi 2008). Equations (2.6) impose a jump in the two components of the tangential stress at the porous-liquid interface that is proportional to the liquid velocity. A formal derivation of the latter condition can be found in Ochoa-Tapia \& Whitaker (1995) and Pokrajac \& Manes (2008). As suggested by Tilton \& Cortelezzi (2008), this condition is rigorously derived from the volume averaging of the momentum and continuity equations around the interface, and hence it is preferred to the one proposed by Beavers \& Joseph (1967), which postulated an unrealistic discontinuity in tangential velocity. As recalled by $(2.5 a)$, the continuity of the tangential velocity is instead preserved in the formulation presented herein. Finally, equations (2.7) set an impermeable bottom wall in the lowest part of the porous region and force the tangential stress to be null therein. This condition replaces the (more rigorous) no-slip condition in order to prevent the inclusion of a gradient in the mean velocity profile at $z=-S$, which would only add complexity to the problem without any real gain in the stability analysis.

\subsection{The eigenvalue problem}

Following the Prandtl-like mapping

$$
\zeta= \begin{cases}\frac{z}{D\left(x_{h}, t\right)} & (z \geqslant 0), \\ z & (z<0),\end{cases}
$$


the porous-liquid interface is mapped to $\zeta=0$, the free surface to $\zeta=1$ and the impermeable bottom to $\zeta=-S$. A linear stability analysis in the three-dimensional domain requires the system to be forced with a generic harmonic disturbance with the normal-mode ansatz

$$
\left\{\boldsymbol{u}_{l, p}, p_{l, p}, d\right\}=\left\{U_{l, p}(\zeta), P_{l, p}(\zeta), 1\right\}+\varepsilon\left\{\boldsymbol{u}_{l, p}^{(1)}(\zeta), p_{l, p}^{(1)}(\zeta), d^{(1)}\right\} \mathrm{e}^{\mathrm{i} \alpha x+\omega t} \cos (\beta y)+\text { c.c. }
$$

(where c.c. is complex conjugate), where $\varepsilon \ll 1, \omega$ is the complex growth rate, $\alpha$ and $\beta$ are the longitudinal and spanwise real wavenumbers, respectively, and $\boldsymbol{u}_{l, p}^{(1)}=\left\{u_{l, p}^{(1)}, \mathrm{i} v_{l, p}^{(1)} \tan (\beta y), w_{l, p}^{(1)}\right\}$ (for brevity, we have omitted the brackets for quantities with subscript ${ }_{p}$ ). In addition, we use the Squire transformations, $k^{2}=\alpha^{2}+\beta^{2}$ and $k \mathscr{U}_{l, p}=\alpha u_{l, p}^{(1)}+\beta v_{l, p}^{(1)}$, to reduce the three-dimensional problem to an equivalent twodimensional problem (e.g. Drazin \& Reid 1981), whereas the use of two (modified) Lagrange functions, $\psi_{l, p}(\zeta)$, which satisfy the continuity equations at the order $O(\varepsilon)$, and which are defined by

$$
\begin{array}{r}
\mathscr{U}_{l}=\psi_{l}^{\prime}+\frac{\alpha}{k} \zeta U_{l}^{\prime} d^{(1)}, \quad w_{l}^{(1)}=-\mathrm{i} k \psi_{l}, \\
\mathscr{U}_{p}=\psi_{p}^{\prime}, \quad w_{p}^{(1)}=-\mathrm{i} k \psi_{p},
\end{array}
$$

reduces the flow field problem to a modified Orr-Sommerfeld problem (the prime symbol refers to the $\zeta$ derivative). One should recall that $\operatorname{Re}[\omega]$ is the growth rate and $c \equiv-\operatorname{Im}[\omega] / k$ is the phase velocity of perturbations. Furthermore, unstable (stable) conditions correspond to $\operatorname{Re}[\omega]>0(\operatorname{Re}[\omega]<0)$ and downstream (upstream) migration of individual waves corresponds to $c>0(c<0)$.

By introducing (2.8) and (2.9)-(2.11) into the governing equations (2.2)-(2.7), at the leading order $O(1)$, we obtain the basic state problem

$$
\begin{gathered}
U_{l}^{\prime \prime}+2=0, \quad P_{l}^{\prime}+\Delta=0, \\
U_{p}^{\prime \prime}-\frac{\epsilon}{\sigma^{2}} U_{p}+2 \epsilon=0, \quad P_{p}^{\prime}+\epsilon \Delta=0,
\end{gathered}
$$

with the boundary conditions

$$
\begin{gathered}
U_{l}^{\prime}=P_{l}=0 \quad(\zeta=1), \\
\llbracket P \rrbracket=\llbracket U \rrbracket=\frac{\sigma}{\epsilon}\left(U_{p}^{\prime}-\epsilon U_{l}^{\prime}\right)-\tau U_{l}=0 \quad(\zeta=0), \\
U_{p}^{\prime}=0 \quad(\zeta=-S),
\end{gathered}
$$

where $\llbracket a \rrbracket=a_{l}-a_{p}$. The analytical solution of the basic state problem (2.12)-(2.16) consequently is

$$
\begin{gathered}
U_{l}=-\zeta^{2}+2 \zeta+\frac{2 \sigma[C(\sigma-\sqrt{\epsilon})-\sqrt{\epsilon}+\sigma]}{C(1+\sqrt{\epsilon} \tau)+\sqrt{\epsilon} \tau-1}, \quad P_{l}=\Delta(1-\zeta), \quad(2.17 a, b) \\
U_{p}=-\frac{2 \sqrt{\epsilon} \sigma(\sigma \tau+1)}{C(1+\sqrt{\epsilon} \tau)+\sqrt{\epsilon} \tau-1}\left(\mathrm{e}^{\sqrt{\epsilon} \zeta / \sigma}+C \mathrm{e}^{-(\sqrt{\epsilon} \zeta / \sigma)}\right)+2 \sigma^{2}, \quad P_{p}=\Delta(1-\epsilon \zeta),
\end{gathered}
$$

where $C=\mathrm{e}^{-2 \sqrt{\epsilon} S / \sigma}$. Assuming that $\sigma \leqslant 0.02, \epsilon=O\left(10^{-1}\right)$ and $S=O(1)$ or larger, it follows that $C \leqslant 10^{-13}$; hence, without any loss in accuracy, the basic state solution of the velocity profile can be simplified to

$$
U_{l}=-\zeta^{2}+2 \zeta-\frac{2 \sigma(\sqrt{\epsilon}+\sigma)}{\tau \sqrt{\epsilon}-1}, \quad U_{p}=\frac{2 \sigma(\sigma \tau+1) \mathrm{e}^{\sqrt{\epsilon} \zeta / \sigma}}{\epsilon^{-1 / 2}-\tau}+2 \sigma^{2} .
$$



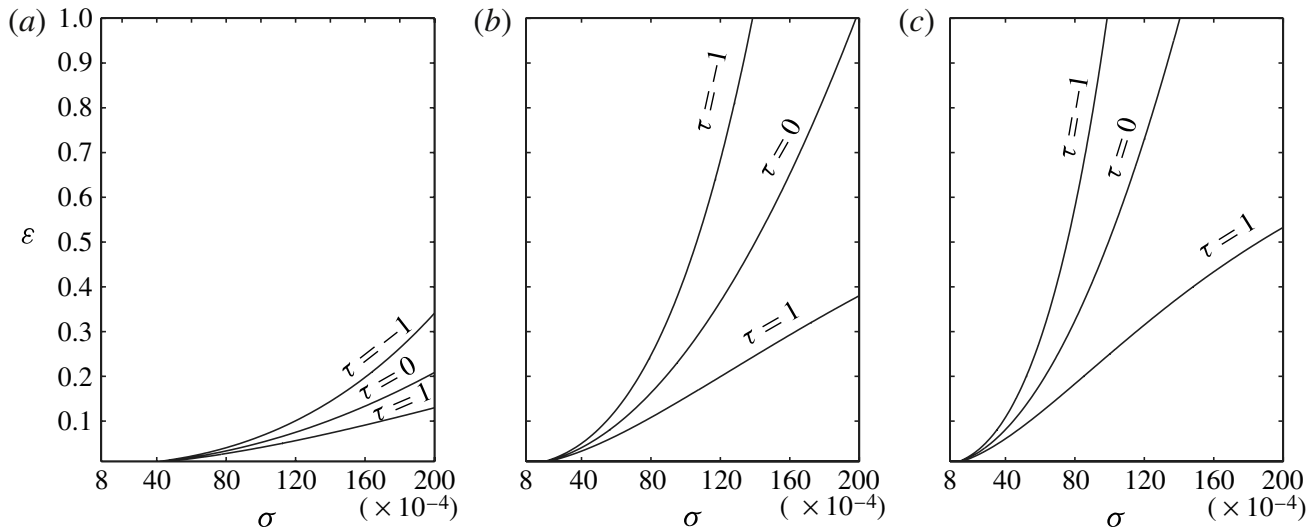

FIGURE 2. Region of validity of the assumption of negligible convective terms in the $\epsilon-\sigma$ plane for: $(a) R e=1000 ;(b) R e=5000 ;(c) R e=10000$. The black lines correspond to the margin $K_{c}=R e$ for different values of $\tau$, while $K_{c}>\operatorname{Re}$ above the lines.

The value of $K_{c}$ is now computed in order to assess the significance of the convective term, as specified above. Henceforth, it is assumed that $R e<10^{4}$ and $\sigma \leqslant 0.02$. The condition $K_{c} \gg R e$ requires an evaluation of the depth, $\delta$, of the Brinkman layer. In principle, the whole porous region is affected by the interface condition, since $U_{p}^{\prime}$ is exactly zero only at the bottom. This suggests that $\delta=S$. However, only in a small part of the porous region is the gradient of the mean velocity profile significant, and therefore a different criterion of evaluation of $\delta$ is required here. An estimation of the Brinkman layer depth can be obtained from the so-called vorticity thickness at the interface, which is defined as $\delta=U_{p}^{\prime}(0)\left[U_{p}(0)-U_{p}(-S)\right]$. By means of this definition and $(2.19 b), K_{c}$ reads

$$
K_{c}=\frac{2 \epsilon(\sigma \tau+1)^{2}}{\sigma^{2}\left(\sigma \epsilon^{-1 / 2}+1\right)\left(1-\tau \epsilon^{1 / 2}\right)} .
$$

Figure 2 reports the behaviour of $K_{c}$ as a function of $\sigma, \epsilon$ and $\tau$. The condition $K_{c}>\operatorname{Re}$ (that is, above each curve) is satisfied in a region of parameter space that shrinks as $R e$ increases. In particular, for $R e=5000$ and 10000 , some cases exist where convective terms have to be retained if a threshold in permeability is exceeded, independently of porosity (e.g. $\tau=-1$ and $R e=5000$, or $\tau=-1,0$ and $R e=10000)$. In order to extend the significance and the impact of the present study, the stability analysis has hence been carried out by retaining the convective terms in the momentum equations. However, there are many gravity-driven flows with significantly low Reynolds numbers where only surface instability is an issue. This includes thin-film flows and certain shallow flows such as debris flows. In such cases, the convective terms are negligible, as also shown by figure 2(a).

At order $O(\varepsilon)$, the following differential generalized eigenvalue problem is obtained

$$
\begin{gathered}
{\left[\mathscr{D}^{2}-\Gamma_{l}\left(U_{l} \mathscr{D}-U_{l}^{\prime \prime}\right)-\omega \operatorname{Re} \mathscr{D}\right] \psi_{l}=0,} \\
{\left[\mathscr{D}^{2}-\Gamma_{p}\left(U_{p} \mathscr{D}-U_{p}^{\prime \prime}\right)-\omega \operatorname{Re} \mathscr{D}-\mathscr{A} \mathscr{D}\right] \psi_{p}=0,}
\end{gathered}
$$


where $\mathscr{D}=\partial_{\zeta \zeta}-k^{2}, \mathscr{A}=\epsilon / \sigma^{2}+2 \sigma^{2} \Gamma_{p}$ and $\left\{\Gamma_{l}, \Gamma_{p}\right\}=\mathrm{i} \alpha \operatorname{Re}\left\{1, \epsilon^{-1}\right\}$. Finally, the boundary conditions (2.4)-(2.7) read

$$
\begin{gathered}
k \psi_{l}+\alpha\left(1+U_{I}\right) d^{(1)}=\mathrm{i} \omega d^{(1)}, \quad k \psi_{l}^{\prime \prime}+k^{3} \psi_{l}-2 \alpha d^{(1)}=0 \quad(\zeta=1), \\
\psi_{l}^{\prime \prime \prime}-\left[3 k^{2}+\Gamma_{l}\left(1+U_{I}\right)\right] \psi_{l}^{\prime}-\Omega d^{(1)}=\omega \operatorname{Re} \psi_{l}^{\prime} \quad(\zeta=1), \\
\llbracket \psi \rrbracket=\llbracket \psi^{\prime} \rrbracket=0, \quad \frac{\psi_{p}^{\prime \prime}}{\epsilon}-\psi_{l}^{\prime \prime}+\frac{\tau}{\sigma} \psi_{l}^{\prime}=0 \quad(\zeta=0), \\
\llbracket \psi^{\prime \prime \prime} \rrbracket=\psi_{l}^{\prime}\left(k^{2}+U_{I} \Gamma_{l}-\frac{\epsilon}{\sigma^{2}}-\alpha k^{2}\right)-2 \Gamma_{l} \psi_{l} \quad(\zeta=0), \\
\psi_{p}=\psi_{p}^{\prime \prime}=0 \quad(\zeta=-S) .
\end{gathered}
$$

In the above equations, the term $\Omega=\mathrm{i} k \operatorname{Re}\left(\Delta+k^{2} / W e\right)$ accounts for the effects of bed inclination and surface tension, whereas $U_{I}=U_{l}(0)=U_{p}(0)$ is the slip velocity at the interface, which can be obtained from $(2.19 a)$ or $(2.19 b)$. Equation (2.21) is the celebrated Orr-Sommerfeld equation, while (2.22) is its extension to the porous case.

Equations (2.21)-(2.27) summarize the physical problem that will be solved in this paper. However, a few remarks are in order. Firstly, it is important to observe that the function $U_{p}$ appears in (2.22) only because the convective term has been retained in the volume-averaged equation (2.3). Otherwise, the velocity profile in the porous region and the structure of the Brinkman layer would have no effects on the Orr-Sommerfeld-like equation in the porous region. This means that, other than affecting the boundary conditions at the interface, any assumed shape of the velocity profile in the porous region (even the flat one) would have been equivalent if the convective terms had not been included in the problem. This opens the possibility to investigate the extent to which the velocity profile in the Brinkman layer affects the stability analysis throughout the domain. Secondly, (2.21) and (2.22) are structurally similar but present some important differences, namely, the presence of the term $\mathscr{A}$ in (2.22), which is associated with the Darcy term, and the difference in the basic state velocity profiles $U_{l}(\zeta)$ and $U_{p}(\zeta)$. These two aspects add mathematical complexity to (2.22) with respect to the classical Orr-Sommerfeld equation. Such an enhanced complexity is circumvented by means of a refined spectral method, which is presented in the following section.

\section{Spectral solution}

The eigenvalue problem was solved by using a spectral-Galerkin method with numerical integration based on the work of Giannakis, Fischer \& Rosner (2009), Camporeale, Canuto \& Ridolfi (2012) and Camporeale \& Ridolfi (2012b), to which the reader can refer for further details. This method is crucial to obtain the whole spectrum of eigenvalues presented and discussed in $\S 4$.

The aim is to recast the generalized differential eigenvalue problem (2.21)-(2.27) in the algebraic form $\boldsymbol{A} \boldsymbol{w}=\omega \boldsymbol{B} \boldsymbol{w}$. The problem is therefore discretized with a spectral-Galerkin technique, which prevents the onset of spurious eigenvalues (e.g. Canuto et al. 2006). A modal representation of the solution is adopted, where the eigenfunctions $\left\{\psi_{l}, \psi_{p}\right\}$ are expanded in the (truncated) spectral form

$$
\psi_{l}=\sum_{i=-3}^{N} \varphi_{i}^{(l)} \Psi_{i}^{(l)}(\xi), \quad \psi_{p}=\sum_{i=-1}^{N} \varphi_{i}^{(p)} \Psi_{i}^{(p)}(\xi),
$$


with $\left\{\Psi_{i}^{(l)}, \Psi_{i}^{(p)}\right\}$ being two sets of trial functions, whereas $\left\{\varphi_{i}^{(l)}, \varphi_{i}^{(p)}, d^{(1)}\right\}=: \boldsymbol{w}$ represents the unknown complex eigentriple. For numerical convenience, the vertical coordinate is further mapped to the range $y \in[-1,1]$ for both phases, according to $\xi=2 \zeta-1$ (for $\zeta>0$ ) and $\xi=2 \zeta / S+1$ (for $\zeta<0$ ). The original eigenvalue problem is then multiplied by a set of test functions, $V=\left\{v \in C^{2}([-1,1])\right\}$, and integrated over the domain $[-1,1]$. The repeated use of integration by part allows the fourth and third derivatives to be reduced to second-order derivatives. Furthermore, the normal component of the dynamic boundary condition (2.24) and the continuity condition on the normal stresses at the interface (2.26) are incorporated in the mass and stiffness operators in the so-called weak form (Canuto et al. 2006) through the boundary term that arises from the integration by parts of (2.21) and (2.22), respectively.

The correct choice of the trial and test functions enables the use of the remaining boundary conditions to be set in a strong form that is equivalent to having additional rows in the ultimate algebraic system. Following Shen (1994), we consider the set of polynomials

$$
\Psi_{i}^{(l, p)}=\sqrt{i+\frac{3}{2}}\left(\frac{\mathrm{L}_{i+3}-\mathrm{L}_{i+1}}{(2 i+3)(2 i+5)}-\frac{\mathrm{L}_{i+1}-\mathrm{L}_{i-1}}{(2 i+1)(2 i+3)}\right), \quad i \in[1, N-3],
$$

where $\mathrm{L}_{i}(\xi)$ denotes the $i$ th Legendre polynomial. These functions are obtained by integrating twice each Legendre polynomial, while enforcing zero boundary conditions at $\xi= \pm 1$ for the function and its first derivative. The use of Lagrange polynomials allows the (troublesome) fourth-order derivative term in the OS equation to be transformed - after twice integrating by parts - into a unitary matrix, thus reducing the risk of round-off, typical of the collocation schemes.

The set of functions in (3.2) is extended by adding other six low-degree polynomials, which accommodate the non-vanishing boundary conditions (2.23), (2.25c) and (2.27). They are:

$$
\left.\begin{array}{l}
\Psi_{-3}^{(l)}=\frac{1}{4}(\xi+1)^{2}, \quad \Psi_{-2}^{(l)}=\frac{1}{4}\left(3-\xi^{2}-2 \xi\right), \\
\Psi_{-1}^{(l)}=\frac{1}{2}\left(1-\xi^{2}\right), \quad \Psi_{0}^{(l)}=\frac{1}{4}\left(2+3 \xi-\xi^{3}\right),
\end{array}\right\}
$$

Finally, the test space for the present problem is conveniently set to $V=$ $\left\{\Psi_{0}^{(l)}, \Psi_{i}^{(l)}, \Psi_{0}^{(p)}, \Psi_{i}^{(p)}\right\}$.

It is worth observing that the test and trial sets are entirely composed by polynomial functions, and therefore the term-by-term integration of (2.21) is analytically tractable. In contrast, because of the exponential structure of $U_{p}(\zeta)$, the integration of coefficients appearing in (2.22) has to be done with a numerical technique. To this end, an interpolatory Legendre-Gauss quadrature formula was employed. This formula approximates a generic integral $I=\int f(\xi) \mathrm{d} \xi$ by the sum $I_{M}=\sum_{j=0}^{M} \varpi_{j} f\left(\xi_{j}\right)$, where $\xi_{j}(j=0, \ldots, M)$ are zeros of $\mathrm{L}_{M+1}$. As far as the weights $\varpi_{j}$ are concerned, several algorithms have been proposed for their computation. Here the approach of Swarztrauber (2002) has been adopted. This approach is based on the Fourier expansion of the Legendre polynomials evaluated for $\xi=\cos \vartheta$ coupled with the Newton-Raphson method to compute their zeros (nearly equally spaced in the $\vartheta$ variable). As shown in Camporeale et al. (2012), this choice minimizes the accumulation of round-off errors for large values of $M$.

The algebraic problem, $\boldsymbol{A} \boldsymbol{w}=\omega \boldsymbol{B} \boldsymbol{w}$, is easily solved by means of the QZ-algorithm. It is emphasized here that the above technique has three fundamental advantages: 

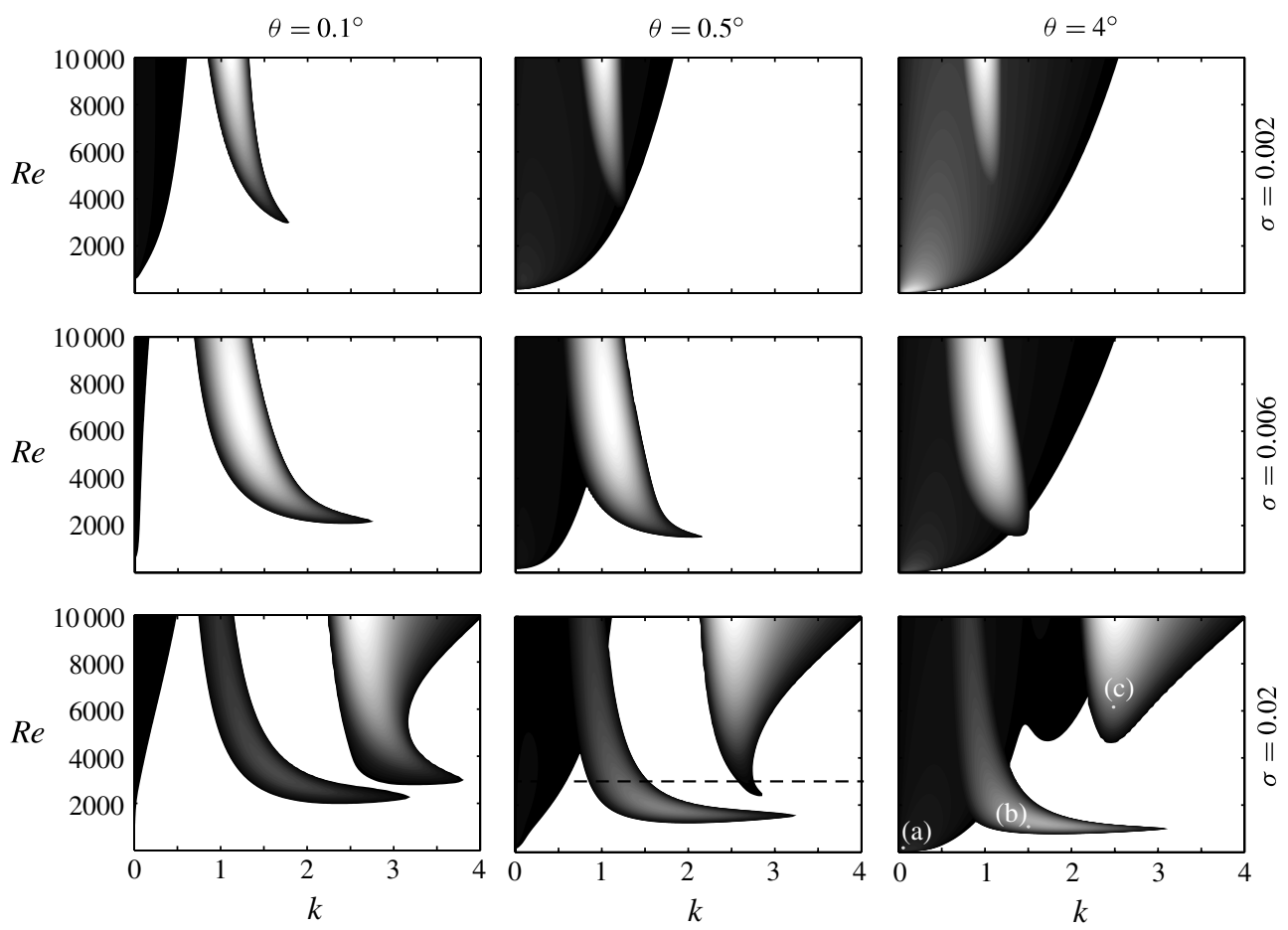

FIGURE 3. Colour maps of the temporal growth rate, in black-to-white scale, in the plane $(k, R e)$, for three different values of the bed inclination, $\theta$, and three different values of permeability, $\sigma$. The uncoloured zone indicates stable conditions $\left(\omega_{r}<0\right)$.

(i) it assures high spectral accuracy and convergence in the solution, provided that the truncation number $N$ is of the order of $O\left(10^{2}\right)$; (ii) it avoids spurious eigenvalues; and (iii) it provides the whole set of eigenvalue spectra and related eigenfunctions.

\section{Results}

The whole analysis has been carried out considering water at standard conditions (i.e. $v=1.7 \times 10^{-6} \mathrm{~m}^{2} \mathrm{~s}^{-1}, \tilde{l}_{c} \sim 2 \mathrm{~mm}$ ) and porous medium with a fixed porosity $\epsilon=0.6$ and a fixed thickness $S=20$. For the interface condition, the coefficient $\tau$ has been set to zero. The results for different sets of these parameters are reported in the supplementary material (available at http://dx.doi.org/10.1017/jfm.2013.16) and will be commented upon later. The independent parameters governing the eigenvalue problem (2.21)-(2.27) hence reduce to $R e, k, \theta$ and $\sigma$. Note that the Froude number is linked to the previous ones via (2.1), through the relationship $2 F r^{2}=\operatorname{Re} \sin \theta$. Two-dimensional perturbations are first considered and hence $k=\alpha$.

Figure 3 shows the results of the stability analysis for $\theta=\left\{0.1^{\circ}, 0.5^{\circ}, 4^{\circ}\right\}$ and $\sigma=\{2,6,20\} \times 10^{-3}$, spanning the ranges $R e \in\left[1-10^{4}\right]$ and $k \in[0-4]$. It is evident that a complex scenario emerges depending on the different parametric conditions.

Looking at figure 3 by moving downwards (i.e. with $\sigma$ increasing), it becomes clear that, at relatively low values of permeability, the domain of instability is characterized by two different patterns, which, at very low slopes, are well separated, but overlap when $\theta$ increases. These patterns correspond to two modes of instability: the surface 
and shear instability. At $\theta=0.1^{\circ}$, the surface mode ( $\mathrm{SuM}$ ) is confined to very low wavenumbers, embraces the whole range of Reynolds numbers ( $\operatorname{ee}_{c}$ is very low), and usually shows lower growth rates than the other modes. The shear mode (ShM) displays higher growth rate but - at low permeabilities - maintains $R e_{c}$ at high values and exists in a small range of wavenumbers. It is interesting to observe that, with the increase in slope, SuM eventually embraces the ShM domain so that the two instabilities coexist in the same region of parameter space.

By increasing the wall permeability, the above scenario modifies in a complex and interesting way. In this respect, three aspects are worthy of mention. Firstly, the ShM domain enlarges, involving lower values of $R e$ and - at very high $\sigma$ - a wider range of wavenumbers, eventually assuming an L-shaped configuration. It follows that, as a consequence of the interactions with the porous medium, a larger band of harmonics is triggered in the film. Secondly, at $\sigma=0.02$ a new kind of porosity-induced instability arises at larger wavenumbers than ShM. We name this the porosity-induced mode of kind $\alpha\left(\mathrm{PIM}_{\alpha}\right)$, in order to distinguish it from other kinds of porosity-induced instabilities, such as the stable one $\left(\mathrm{PIM}_{\beta}\right)$ found by Tilton \& Cortelezzi (2008) in channel flows, and the unstable one $\left(\mathrm{PIM}_{\gamma}\right)$ detected by Liu \& Liu (2009) neglecting all the inertial terms in the equation of motion within the porous medium. PIM $\mathrm{P}_{\alpha}$ is absent in both the impermeable case and closed-channel flows, and it is triggered by the coupling between the film flow and the porous flow through the interface. This suggests that the presence of the free surface triggers new mechanisms inside the porous region. It is also worth noting that the maximum growth rate of $\mathrm{PIM}_{\alpha}$ is usually higher than for the other modes, and the shear and porous domains are very close at small $\theta$ and $k \sim 3$. Finally, the major complexity is displayed at high values of $\theta$ and $\sigma$ : mutual interactions between the three modes arise, the domain of instability being overlapped in two zones. Moreover, at intermediate values of $k$ (in the range 1.5-2.5), these interactions induce a slight change in the marginal stability curve of the SuM, thus generating a second relative minimum, which is otherwise absent in the impermeable case. This aspect is quite important since it is the symptom that permeability also affects the free-surface dynamics, besides the core of the film dynamics. It turns out that mutual interactions are activated between the porous zone and the free surface, a point that will be further discussed in $\S 5$. The wall permeability leads the system to a state where different kinds of instability coexist and interact with each other: for instance, the dashed line, drawn at $R e=3000$, crosses the SuM (low wavenumbers), ShM (intermediate wavenumbers) and $\mathrm{PIM}_{\alpha}$ (high wavenumbers) domains.

It is now instructive to move deeper into the description of the eigenvalue set and the corresponding eigenfunctions related to the above-mentioned three modes of instability. To this end, three conditions are marked in the bottom right panel of figure 3: (a) SuM, (b) $\mathrm{ShM}$ and (c) $\mathrm{PIM}_{\alpha}$. The results are depicted in figure 4, where the panels on the left-hand side report the corresponding spectra in the growth rate-phase velocity plane: black dots refer to the present problem, while the green dots show the outcome of a standard problem of film flow over an impermeable wall (i.e. the same parameters but $\sigma=0$ ). Arrows indicate the least-stable eigenvalue, its abscissa being the growth rate reported in figure 3. As expected, the overall structure of the SuM spectrum (figure $4 a(\mathrm{i})$ ) is weakly affected by the presence of a porous bed and the green and black dots are indeed very close. In contrast, the eigenvalue response of the ShM (figure $4 b(\mathrm{i})$ ) is somewhat different from the impermeable problem. The well-known three-branch structure of the Orr-Sommerfeld spectrum (Grosch \& Salwen 1968), reported by green dots, is in fact altered in the 
(a)

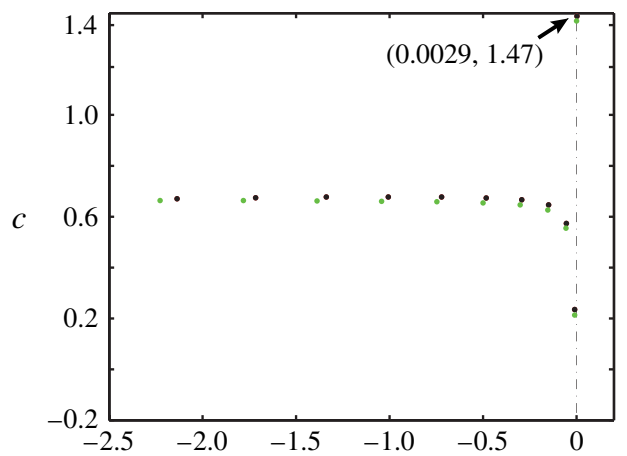

(b)

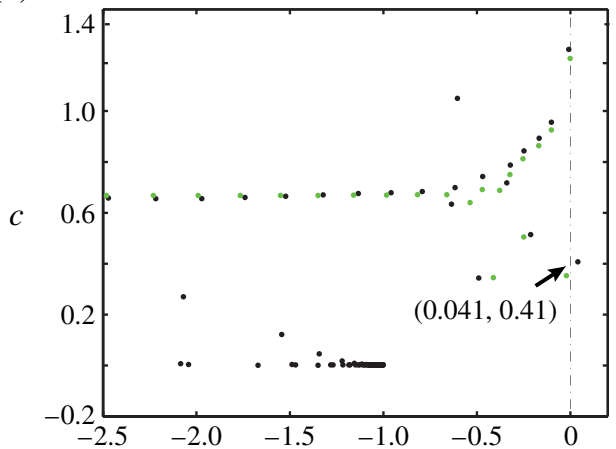

(c)

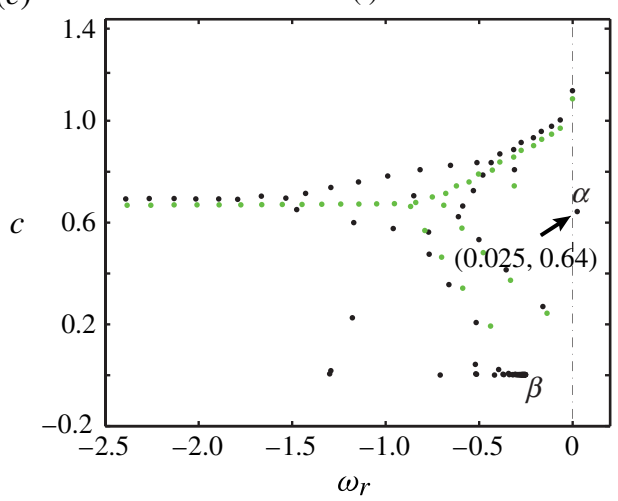

(ii)

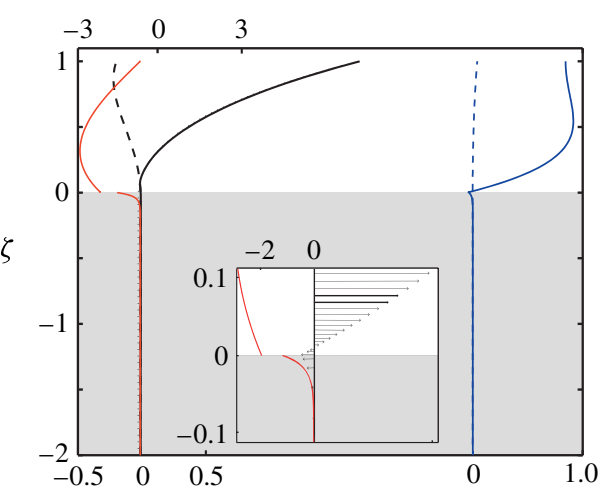

(ii)

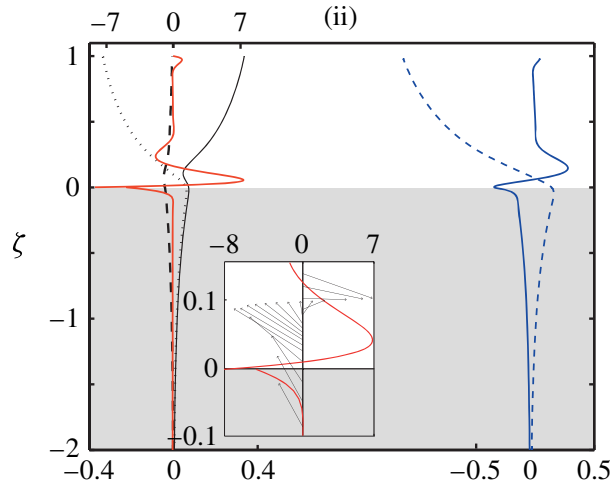

(ii)

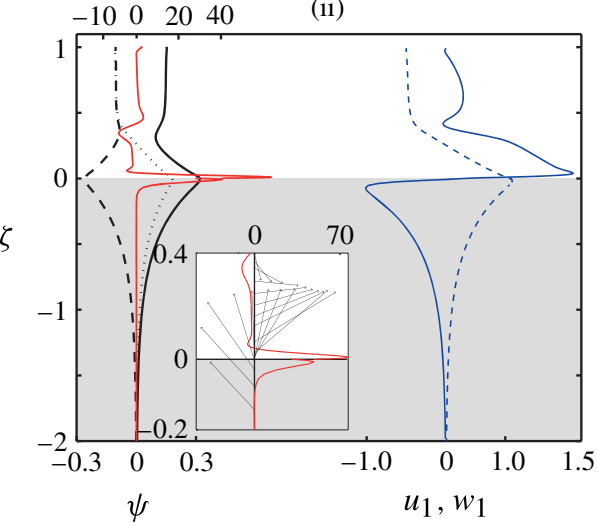

FIGURE 4. Spectra (i) and eigenfunctions (ii) of the marked eigenvalues, for the three type of instabilities: (a) SuM (referring to point a in figure 3), (b) ShM (point b in figure 3) and (c) PM (point $\mathrm{c}$ in figure 3). Green dots refer to the spectra of the corresponding impermeable case. Black curves: $|\psi|$, solid line; $\operatorname{Re}[\psi]$, dashed line; $\operatorname{Im}[\psi]$, dotted line. Blue curves: $u^{(1)}$, solid line; $w^{(1)}$, dashed line. Red curve: vorticity perturbation, $\phi$. The insets show a zoom of the flow field (arrows) and vorticity at the porous-liquid interface. Note the different scales of the $\phi$ and $\psi$ axes in panels (ii). 
proximity of the divide of its so-called $\mathrm{S}$ branch. As is well known, this branch corresponds to wavelets advected at the mean velocity of the film, as confirmed by their value of the phase velocity, i.e. $c \sim 2 / 3$. Such a change is probably due to the influence of a novel porosity-induced double-branched set of eigenvalues that occurs at very low phase velocity.

This modification of the standard picture is exacerbated in the conditions that are favourable to trigger the $\mathrm{PIM}_{\alpha}$ instability (figure $4 c(\mathrm{i})$ ), where the mentioned interactions between the two parts of the spectrum give rise to a new 'unstable' eigenvalue that lacks a counterpart in the standard Orr-Sommerfeld spectrum (marked with $\alpha$ ). Note also that, although such an eigenvalue is triggered by the porous medium, it exhibits a phase velocity very close to the $\mathrm{S}$ branch, which means that the corresponding wave is locked to hydrodynamic instabilities in the free flow. This suggests that $\mathrm{PIM}_{\alpha}$ is intrinsically related to the film hydrodynamics and it is dynamically different from the other modes that are set on the lower porosity-induced branch at $c \sim 0$, which are invariably stable. These latter modes are instead equivalent to the $\mathrm{PIM}_{\beta}$ identified by Tilton \& Cortelezzi (2008).

Figure $4(a-c)($ ii) report the eigenfunction $\psi$ corresponding to the least-stable eigenvalues of figure $4(a-c)$ (i) (black curves), the related flow fields (blue curves) - as computed by (2.10) and (2.11) - and the perturbations of vorticity $\phi$ (red curves). The choice $x=0$ is made without loss of generality. The eigenfunctions have been normalized in order to impose a unitary kinetic energy onto the flow field, namely

$$
\begin{aligned}
& \int_{-S}^{0}\left|u^{(1)}\right|^{2}+\left|w^{(1)}\right|^{2} \mathrm{~d} \zeta \\
& \quad=2 \int_{-S}^{0} \operatorname{Re}\left[\psi_{p}^{\prime}\right]^{2}+k \operatorname{Im}\left[\psi_{p}\right]^{2} \mathrm{~d} \zeta+2 \int_{0}^{1} \operatorname{Re}\left[U_{l}^{\prime} \zeta d^{(1)}+\psi_{l}^{\prime}\right]^{2}+k \operatorname{Im}\left[\psi_{l}\right]^{2} \mathrm{~d} \zeta=1 .
\end{aligned}
$$

As known, vorticity is of paramount importance for the development of fluid instability. Given its relationships with velocity gradients, it diffuses from the boundaries, where its production is highest, throughout the whole flow domain. Vorticity is here obtained through the relationship $\phi=\psi^{\prime \prime}-k^{2} \psi-2 d^{(1)} \zeta \mathscr{H}(\zeta)+$ c.c., where $\mathscr{H}(\zeta)$ is the Heaviside function and symbol $\psi$ is intended with subscript $l(p)$ for positive (negative) values of $\zeta$. After manipulating (2.10a), (2.19a) and (2.25) one gets

$$
\llbracket \phi \rrbracket=2(1-\epsilon)\left[T_{r} R e+\alpha w_{i}+d_{r}^{(1)}\right]_{\zeta=0^{+}},
$$

where $T_{r}$ is the real part of the liquid shear stress perturbation, $w_{i}=\operatorname{Im}\left[w_{l}^{(1)}\right]$ and $d_{r}^{(1)}$ is the real part of the flow depth perturbation. From (4.2) one notes that, although a no-jump stress condition is employed here (i.e. $\tau=0$ in (2.6)), vorticity is continuous over the liquid-solid interface only in the limiting case $\epsilon \rightarrow 1$ (namely, $l_{s} \sim 0$ ). It should also be remarked that the vorticity jump is affected by the Reynolds number, the flow flux through the interface and depth perturbation.

Keeping these preliminary remarks in mind, figure $4(a-c)($ ii) are worth pointing out as they provide information on the flow field generated by each type of instability and complement the interpretation of results already obtained from the eigenvalue spectra. The SuM and the ShM appear as centre and wall modes, respectively. The flow field structure of SuM is relatively simple, with very low vertical components (blue dashed line) and maximum velocity in the upper part of the domain. In this case, the jump in the vorticity at the interface is very low $(\llbracket \phi \rrbracket=-0.9)$ and its maximum absolute value is $|\phi|_{m}=3$. In contrast, the ShM eigenfunction is structurally very complex, represented by a flow field with significant vertical velocities and 


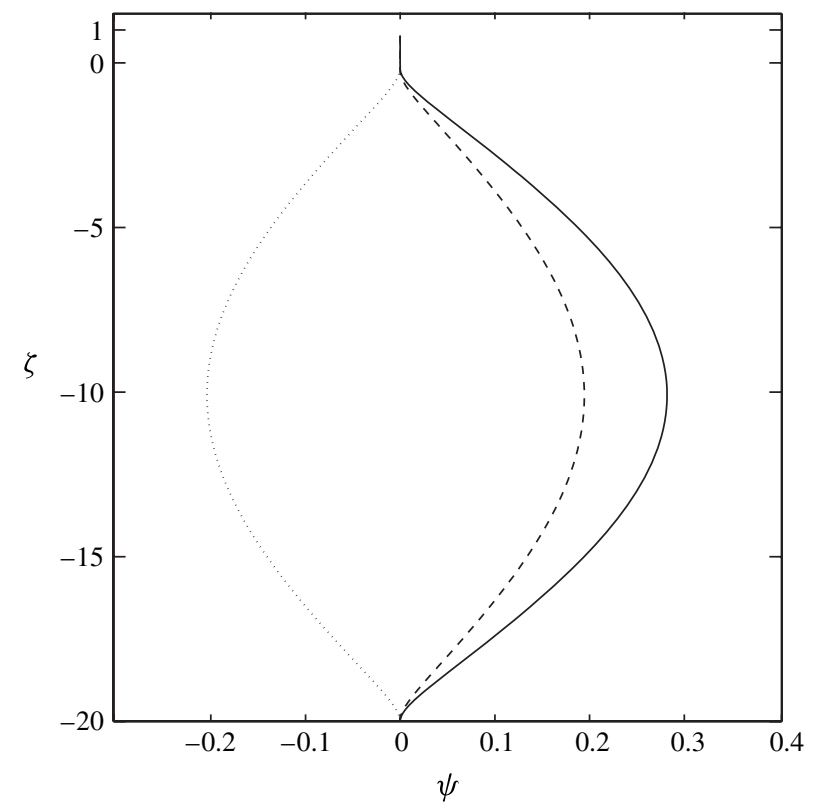

FIGURE 5. Eigenfunction of $\operatorname{PIM}_{\beta}$ indicated in figure 4(c)(i): $|\psi|$, solid line; $\operatorname{Re}[\psi]$, dashed line; $\operatorname{Im}[\psi]$, dotted line.

high vorticity intensity, with several changes in the sign and $\left\{\llbracket \phi \rrbracket,|\phi|_{m}\right\}=\{-3.35,7\}$. However, despite the presence of the porous medium, the eigenfunctions of the ShM closely resemble the well-known Tollmien-Schlichting instabilities commonly observed in wall flows (Schmid \& Henningson 2001). The eigenfunction of the $\mathrm{PIM}_{\alpha}$ (figure $4 c$ (ii)) exhibits a higher degree of variability and intensity: the vertical velocity component reaches the maximum value at the interface, where, combined with the high values of $R e=6000$ and $k=2.5$ (see (4.2)), it induces a very large jump in vorticity: $\llbracket \phi \rrbracket=15.65$. Furthermore, again at the interface, we observe the maximum vorticity intensity $\left(|\phi|_{m}=65\right)$, an order of magnitude larger than the other modes. Finally, it is worth noting that the response of the flow within the porous region is dramatically different for the three modes. While SuM perturbations are nearly absent for $\zeta<0$, the ShM shows a weak propagation of the instability in the upper part of the porous zone, coincident with the Brinkman layer (see the inset of figure $4 b$ (ii) obtained for $\delta=4 \sigma \epsilon^{3 / 2}=0.03$ ). The $\mathrm{PIM}_{\alpha}$ instead induces perturbations that propagate deeper within the porous medium (i.e. down to $\zeta=-2$ ), probably as an effect of a diffusion-driven mechanism driven by the high vorticity occurring at the interface.

From the above analysis, it turns out that the dynamics at the porous medium-liquid interface is crucial to understand the mechanisms of generation of the instability, where a key role is played by the vorticity generation, the exchanged flux between the two media, along with the long-range interactions with the free surface. We stress that without the latter effect the $\mathrm{PIM}_{\alpha}$ instability does not appear.

We have remarked that, although $\mathrm{PIM}_{\alpha}$ is triggered by the presence of the porous medium, it is intrinsically related to the hydrodynamic instabilities, both dynamically and kinematically. For comparison, figure 5 reports the eigenfunction of $\mathrm{PIM}_{\beta}$, namely 

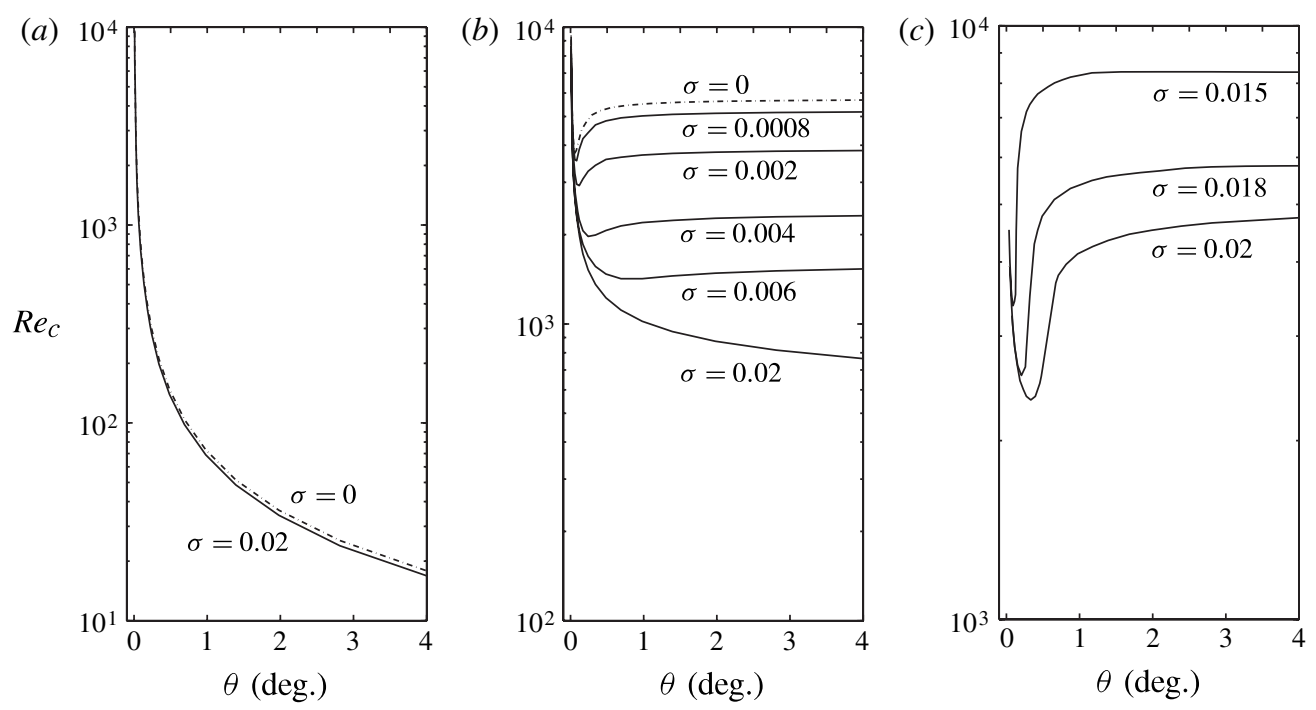

FIGURE 6. $R e_{c}$ versus $\theta$ for different values of permeabilities: (a) SuM; (b) ShM; (c) $\mathrm{PIM}_{\alpha}$.

the rightmost dot of the eigenvalues located at $c \sim 0$ in figure $4(c)(\mathrm{i})$, which are all invariably stable. It is evident that this kind of modal response is purely porous in the sense that it is null on the walls and maximum in the middle of the porous region. It is therefore uniquely dependent on the ordinary differential equation (2.22) and weakly affected by the interface and free-surface dynamics.

The present overview of results is now completed with the analysis of figure 6, which reports the minimum value of the Reynolds number, $R e_{c}$, of the marginal stability curves for the three modes of instability, while spanning several values of slopes and permeabilities. The critical Reynolds number of the SuM instability is not sensitive to permeability. The $\operatorname{Re}_{c}-\theta$ curve almost coincides with the Benjamin-Yih theoretical prediction, regardless of $\sigma$. Stronger permeability effects are instead observed on the other modes of instability. The ShM performs a characteristic minimum in the $\operatorname{Re}_{c}-\theta$ curve at low permeability, and the value of $\operatorname{Re}_{c}$ assumes the standard closed-channel prediction at high slopes (i.e. $R e_{c} \sim 5770$; e.g. Schmid \& Henningson 2001). The presence of a minimum at $\theta=0.05^{\circ}$, for $\sigma=0$, has already been reported in the previous literature without any physical justification, but the underlying mechanism can be interpreted as a competition between two $\theta$-dependent processes, which will be discussed in the following section. With the increase in $\sigma$, the minimum disappears and eventually the curves begin to follow a monotonic decreasing shape. It is therefore confirmed that permeability destabilizes the ShM, thus anticipating the transition to turbulence. As far as the $\mathrm{PIM}_{\alpha}$ is concerned, a minimum value of $R e_{c}$ is invariably exhibited at increasing slopes with permeability, shifting from the coordinates $\left\{\theta, \operatorname{Re}_{c}\right\}=\left\{0.085^{\circ}, 3362\right\}$ at $\sigma=0.015$ to $\left\{0.65^{\circ}, 2228\right\}$ at $\sigma=0.02$. The great resemblance between the shape of the curves associated with $\mathrm{ShM}$ and $\mathrm{PIM}_{\alpha}$ (figure 6b,c) further confirms the relationship between the shear and the porous modes, as discussed above.

In the next section, a physical interpretation of the above results will be proposed. 

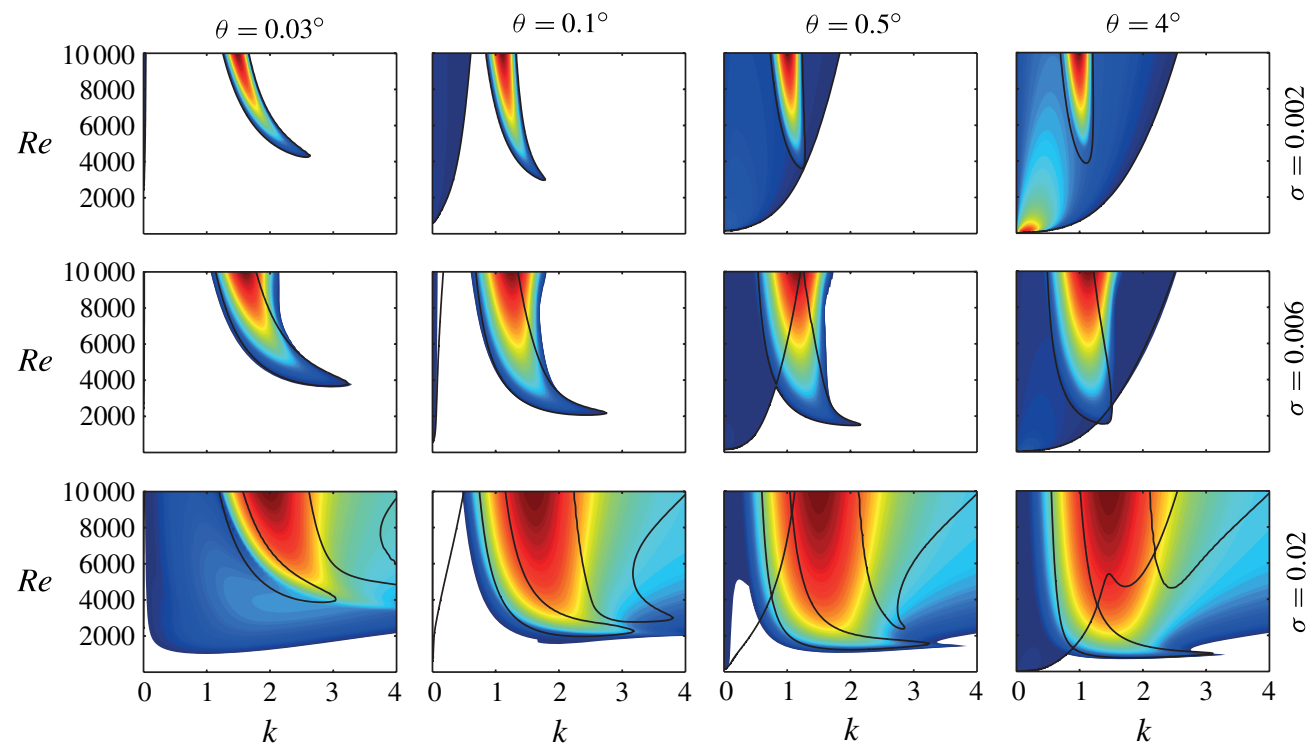

FIGURE 7. Colour maps of the temporal growth rate in blue-to-red scale, in the plane $(k, R e)$, for four different values of the bed inclination, $\theta$, and three different values of permeability, $\sigma$. White zone indicates stable conditions $\left(\omega_{r}<0\right)$. These results have been obtained by removing the convective terms in the momentum balance equation governing the flow within the porous medium; black lines represent marginal stability curves obtained with convective terms.

\section{Discussion}

\subsection{Analysis of the key processes}

The above findings open the door to a number of considerations. Firstly, it is instructive to shed light on the role played by convective terms of the momentum balance equation within the porous region, which have been retained in (2.3) via the term $\epsilon^{-1}\left\langle\boldsymbol{u}_{p}\right\rangle \cdot \nabla\left\langle\boldsymbol{u}_{p}\right\rangle$. It has been mentioned previously that, if this term is neglected (the so-called Stokes approximation), the linearization procedure loses information about the velocity profile of the basic state. The fluid dynamics of the Brinkman layer is therefore oversimplified. This may explain some of the results presented by Liu \& Liu (2009), who suggested that the problem of film flow over a porous medium can be approached using a one-sided model where the effects of the porous region can be reduced to a boundary condition at the interface. Indeed, according to the present study, that result is biased by the fact that it was obtained from a comparison between one-sided simulations and two-sided simulations where the convective terms were not included. This approximation has definitely muted the effects of the porous medium on the linear instability analysis.

A more in-depth analysis on the role played by the convective term in this instability contest is in order. Figure 7 reports temporal growth rates obtained by neglecting the convective terms, and it can be directly compared with figure 3 , where the results from the complete model are presented; for clarity, marginal stability curves of figure 3 are also reported in figure 7 with black lines. Additionally, with respect to figure 3 , figure 7 reports a further column of panels investigating the case of $\theta=0.03^{\circ}$. The comparison between the two figures suggests the following three points. (i) In figure 7 , 
the porosity-induced mode $\mathrm{PIM}_{\alpha}$ is completely suppressed. (ii) At very low slopes and with high permeability, a novel porosity-induced mode appears (see the blue region in the bottom left panel), which can be reasonably associated with the PIM $_{\gamma}$ already detected by Liu \& Liu (2009). (iii) The ShM changes significantly with respect to the complete model: at high permeability it explodes by covering a very large region of the $k-R e$ plane. To summarize, it is evident that the Stokes approximation has important effects on the shear and porous modes, especially for walls with high permeability. The shear mode becomes over-excited, whereas the porous mode switches from the $\alpha$ to the $\gamma$ type and occurs only for low inclination angles. It should be noted that PIM $_{\gamma}$ is also detected by the complete model (not shown here), namely at $R e=O\left(10^{4}\right)$. However, at these Reynolds numbers, the reliability of linear stability analysis becomes questionable and therefore no further comments are made on this result.

The results obtained from the linear stability analysis are now discussed from a more physical point of view. We will focus attention on the physics underlying the occurrence of the ShM and PIM because the surface mode has already been deeply investigated in the previous literature (e.g. see the paper by Kelly et al. (1989) for an interesting energetic justification thereof) and, apparently, it seems to be insensitive to wall permeability. As far as the shear instability is concerned, it is recalled that the 'sag'-like curve for the critical Reynolds number plotted versus the slope, in the case $\sigma=0$, has already been documented in the literature, but a physical justification has never been proposed. One can reasonably assume that a curve that displays a decreasing and an increasing trend is determined by two competing mechanisms and that the minimum represents the cross-over that discriminates the ranges of slopes where one mechanism dominates over the other. Such mechanisms can be intuitively guessed from figure $6(b)$ by following an imaginary line of constant $R e_{c}$, located above the minimum and below the asymptotic value reached at high slopes by the curve representing the impermeable wall case. Within this range of $R e$, the line crosses the $R e_{c}$ curve at two points. The first determines the slope where the associated flow becomes unstable and the second the slope at which the flow becomes stable again. It is proposed that the switch from the unstable to the stable regime is governed by the following two mechanisms. (i) On the one hand, while the slope is increasing, the flow depth decreases and the free-surface velocity increases (i.e. $R e$ is constant). This leads to an increase in shear and hence of vorticity at the wall. (ii) On the other hand, while the depth decreases, the free surface becomes closer to the wall and the damping of stresses imposed by the boundary conditions at the free surface affects in some way the dynamics near the wall. The former mechanism is destabilizing and prevails at very low slopes, whereas the latter is stabilizing and prevails at larger slopes, with the cross-over occurring at a specific value $\theta_{m} \sim 0.05^{\circ}$, where the sag curve shows a minimum (see the dash-dotted line in figure $6 b$ ).

When the interface is porous, a novel mechanism adds to the above scenario. The perturbations on the free surface (either stable or not) hydrostatically induce head differences at the liquid-solid interface, which in turn trigger a flow exchange through the interface itself. The so-generated vertical momentum is therefore longitudinally convected in the porous region via the term $w_{p}^{(1)}(0) U_{p}^{\prime}(0)=2 \epsilon w_{p}^{(1)}(0)$; this convective transport produces vorticity. The vorticity diffuses up in the liquid phase and further destabilizes the shear mode. It follows that with the increase in permeability, the rising of the sag curve at higher slopes induced by the previously mentioned stabilizing process is gradually overwhelmed by the destabilizing effect induced by the flow-exchange mechanism. This leads the $\operatorname{Re}_{c}-\theta$ relationship to follow a monotonic 
behaviour at large permeabilities (solid lines in figure $6 b$ ). The high level of vorticity inferred by the eigenfunctions reported in figure 4 is consistent with this argument.

The same process also has important effects on the porous mode. As we have previously remarked, the $\mathrm{PIM}_{\alpha}$ is essentially an interface mode, which, like the ShM, is induced by vorticity production at the interface. The two modes are also kinematically locked, since we observed that they travel at the same phase velocity $c \sim 2 / 3$. More precisely, such a velocity is not associated with the least stable ShM but with the $\mathrm{P}$ branch of its spectrum (see figure $4 c(\mathrm{i})$ ). In other words, $\mathrm{PIM}_{\alpha}$ is a sibling of ShM, but in order to observe it at physical values of $R e$, a threshold in $\sigma$ has to be passed.

A heuristic computation can show, with further detail, the linkage between the free-surface-induced pumping mechanism through the interface and the vorticity generation in the Brinkman layer. Combining the continuity equation and Darcy's law in the porous region, it is straightforward to show that, at the interface, $\psi_{p}=\mathrm{i} w_{p} / \alpha \sim-\mathrm{i} \alpha \sigma d^{(1)}$, namely, the flux through the interface is proportional to the depth perturbation. In addition, after considering (2.22) at $\zeta \rightarrow 0$ (i.e. imposing $U_{p}=U_{I}$ and $\left.U_{p}^{\prime \prime}=2 \epsilon^{3 / 2} / \sigma\right)$ and remembering that $\phi_{p}=\psi_{p}^{\prime \prime}-\alpha^{2} \psi_{p} \equiv \mathscr{D} \psi_{p}$, one obtains a simplified form of the conservation equation for the vorticity:

$$
\phi_{p}^{\prime \prime}-\left(\Gamma_{p} U_{I}+\alpha^{2}+\mathscr{A}+\omega R e\right) \phi_{p}=-2 \alpha^{2} R e \epsilon^{1 / 2} d^{(1)} .
$$

It suffices here to focus attention on the dominant balance of (5.1) for $\zeta \rightarrow 0$ (cf. Bender \& Orszag 1978). With this aim let us resize the vertical coordinate as $\zeta \rightarrow \delta^{-1} \zeta$, where $\delta=4 \epsilon^{3 / 2} \sigma \sim \sigma$. In this way we obtain

$$
\phi_{p}(0) \sim-\frac{2 \alpha^{2} \epsilon^{1 / 2} d^{(1)}}{\mathrm{i} \alpha U_{I} \epsilon^{-1}+\epsilon \operatorname{Re}^{-1} \sigma^{-2}+\omega},
$$

where the approximations $\delta^{2} \mathscr{A} \sim \delta^{2} \epsilon \sigma^{-2}$ and $\alpha^{2} R e^{-1} \sim 0$ have also been used. Expression (5.2) confirms that vorticity production at the interface is proportional to the free-surface perturbations. Furthermore, by imposing the critical condition $\omega=0-\mathrm{i} \alpha c \sim-2 \mathrm{i} \alpha / 3$ in (5.2), after some algebra, one obtains that $\left|\phi_{p}\right| \rightarrow \infty$ and $\operatorname{Arg}\left[\phi_{p}\right] \sim \operatorname{Arg}\left[d^{(1)}\right]$ for $\sigma \sim \epsilon / 3\left(1+\epsilon^{1 / 2}\right)$. The latter result provides the resonance condition between the forcing of the free surface and the PIM $_{\alpha}$ instability, where the porous region is invariably unstable. Although, with the value of porosity used herein, the resonant value of permeability is $\sigma=0.11$, which is beyond the range of validity of the present formulation, the above derivation is a theoretical confirmation that the porous mode is destabilized by any increase in wall permeability.

It is also remarked that a key element of the 'pumping' mechanism is the convection within the porous region, without which the vertical momentum pumped by the film is only transported (and dissipated) by diffusion. In this case, (5.1) lacks the forcing term on the right-hand side, and thus vorticity at the interface is not externally induced by the vertical fluxes. It is worth recalling that the same result would be obtained by considering the inertial terms but assuming a flat basic state in the porous region. On the contrary, neglecting the convection in the porous region would only further destabilize the ShM (see figure 7). We suspect that this aspect is related to the effect of suction and blowing in the boundary layer, which, as suggested by Tilton \& Cortelezzi (2008), triggers the wall turbulence but, when coping also with the vorticity production from the pumping, is slightly dampened. This aspect requires further investigations. 
Another important point arising in the permeable case is the presence of a slip velocity, which is strictly dependent on the parameter $\tau$. An additional set of simulations (reported in the supplementary material) investigates the role of $\tau$. In summary, since $U_{I}$ increases with $\tau$, high values of this parameter reduce the shear of the basic state and therefore are stabilizing on both ShM and PIM $_{\alpha}$. In particular, the latter instability is completely inhibited for $\tau=1$ (in agreement with the results by Tilton \& Cortelezzi (2008)). The opposite trend is observed for negative values of $\tau$.

Finally, the role of the porous medium thickness $S$ has been investigated through a further set of simulations for values $S=\{0.2,2,5,10,30\}$ (plots are reported in the supplementary material). As far as $\mathrm{PIM}_{\alpha}$ and $\mathrm{ShM}$ are concerned, almost no changes occur if $S$ is large enough to allow flow field perturbations in the porous medium to vanish at a certain distance from the interface that is also well above the impermeable wall (say, $S \gg \delta$ ). This holds for all the investigated values of $S$, except for the smaller one, $S=0.2$. This is in agreement with the outcomes by Tilton \& Cortelezzi (2008), who observed that variations of $S$ in a range that allows the former condition to be satisfied have only weak effects on the perturbations of the normal velocity at the interface. Conversely, a further decrease of $S$ (see $S=0.2$ ) entails a marked reduction in the normal velocity perturbation, which is a stabilizing mechanism: for $\sigma=\{0.002,0.006\}$ a shrinkage of ShM domain and an increment in $R e_{c}$ occur. Additionally, varying $S$ also affects SuM, which undergoes changes in both $R e_{c}$ and the shape of the marginal stability curves as already observed by Liu \& Liu (2009). This latter aspect is particularly evident at low values of bed slope, because of the sensitivity of the base pressure at the bottom to the slope and its interaction with the flow field perturbation in both liquid and porous domains (e.g. (2.17b) and (2.27)).

\subsection{Three-dimensional effects}

We conclude this section with a brief analysis of three-dimensional effects. Squire's theorem, a classic result of hydrodynamic instability theory, states that parallel shear flows become unstable to two-dimensional perturbations (i.e. with $\beta=0$ ) at a value of $R e$ smaller than any other $R e$ value for which three-dimensional unstable perturbations exist. In the analysis of the standard Orr-Sommerfeld problem, it is therefore sufficient to study two-dimensional perturbations (Drazin \& Reid 1981).

The extension of Squire's theorem to open-channel flows was first addressed by Yih (1955), whose approach is formally followed in the present problem too. However, the term $\alpha k^{2} \psi_{l}^{\prime}$, appearing in the normal stress continuity equation (2.26) at the interface, prevents one from finding a set of suitable transformations for $\operatorname{Re}, \theta$ and $\sigma$ that would extend Squire's theorem to the considered context. This is further confirmed by the results reported in figure 8: for sufficiently high values of permeability, it occurs that some three-dimensional unstable disturbances exist (namely, $\alpha$ and $\beta$ are not both null) while the corresponding two-dimensional perturbation is stable for both the reported $R e$ and any other smaller $R e$ value (see also figure 3). It is also remarkable that only the porous mode escapes Squire's theorem predictions, in the investigated range of parameters. This suggests that Yih's conclusions herein hold for ShM and SuM.

From a more physical point of view, it is also interesting to observe that, for high values of the spanwise wavenumber, $\beta$, the range of the unstable longitudinal wavenumbers, $\alpha$, is increased with respect to the case $\beta=0$. The domain of instability of ShM and $\mathrm{PIM}_{\alpha}$ are in fact closer to each other and a larger number of harmonics are therefore excited. However, it should be noted that, despite this disagreement with Squire's theorem, for a fixed set of $R e, \sigma$ and $\theta$, the higher values of the growth rate 

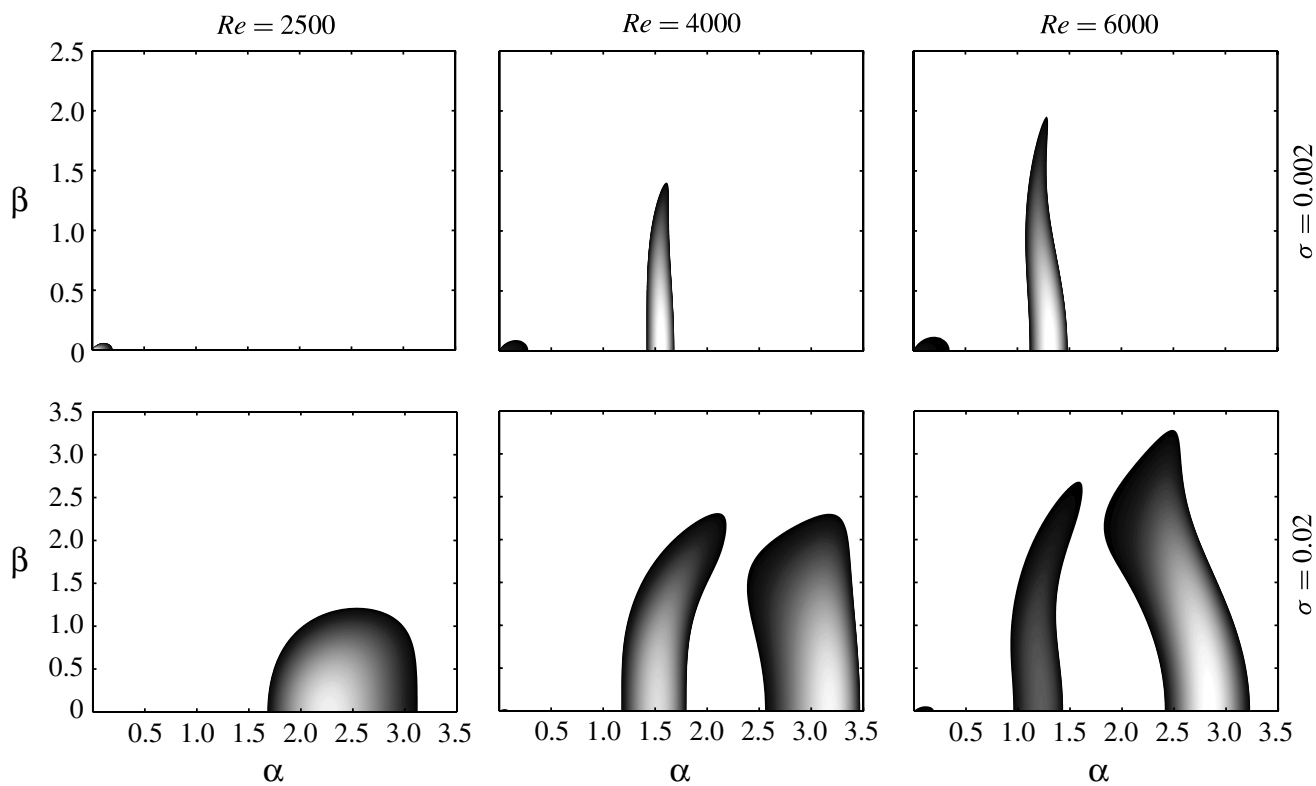

FIGURE 8. Three-dimensional effects: colour maps of the temporal growth rate in black-towhite scale, in the $\alpha-\beta$ plane, for different values of $R e$ and $\sigma$. The uncoloured zone indicates stable conditions $\left(\omega_{r}<0\right)$. $\left[\theta=0.1^{\circ}\right]$.

are attained at $\beta=0$, which suggests that the predominant instability is substantially two-dimensional.

\section{Conclusions}

The present work sheds light on the interplay among the different kinds of instabilities occurring when a gravity-driven film flows over a permeable wall. As known, the literature has dealt with the case of impermeable walls in depth whereas the case of permeable walls has been largely disregarded. However, for both cases, some features of the obtained numerical results still lack a physical justification. For instance, a physical explanation for the occurrence of the well-known 'sag' curve in the $\operatorname{Re}-\theta$ plane has never been proposed.

By means of a novel spectral-Galerkin numerical method, able to provide the whole eigenvalue and eigenfunction spectra with high accuracy, we have tackled the linear stability analysis of a two-sided model, based on the volume-averaged Navier-Stokes equations and with Whitaker's stress-jump conditions at the liquid-solid interface. Furthermore, the convective terms in the porous region were retained because it was shown that they are crucial to obtain the coupling between the basic state and the velocity perturbation in the Brinkman boundary layer. The results have permitted the occurrence of three different modes of instability to be distinguished and discussed: the surface mode, the shear mode and one kind of porosity-induced mode. In particular, the latter was shown to be strictly related to the second one through the action of the interface, but the surface mode also appeared to develop long-distance interactions with the other two modes. Indeed, the porosity-induced mode of type $\alpha$ is not present in the closed-channel problem. Other kinds of porous induced modes - called $\beta$ and $\gamma$ type - were identified in previous works, but we have shown 
that they have no particular significance when the effects of convective terms in the porous region are correctly accounted for. A sensitivity analysis has in fact elucidated that, if the convective terms are neglected, the porosity-induced mode is inhibited, the shear mode is favoured and, for low slopes, a fictitious $\operatorname{PIM}_{\gamma}$ appears with $\operatorname{Re}_{c}$ lower than the shear mode (see bottom left panel in figure 7). This latter aspect suggests that any prediction of transition to turbulence is erroneously underestimated if convective terms are neglected. A rationale based on two competing mechanisms has been proposed to interpret the 'sag'-like curve of the shear mode, whereas the head-induced pumping phenomenon through the interface was shown to explain the dynamics under permeable conditions. Finally, it turns out that Squire's theorem is not entirely applicable to the present problem, even though the most likely perturbations are still two-dimensional.

From the present analysis, two overall considerations emerge. Firstly, we observed that the decrease in $R e_{c}$ due to an increase in permeability is much more evident for the porous mode than for the shear mode. Hence, one could reasonably guess, for permeabilities larger than the ones investigated here and provided a parametrization for the Forchheimer term is available, that the marginal curve of the porosity-induced mode becomes lower than the shear mode, and consequently the transition is therefore initiated by the former one. Secondly, it is well known that a nonlinear stability analysis and/or a non-normal linear stability analysis probably allow lower values for the critical conditions than a standard linear stability analysis. Because the critical conditions of the shear mode and the porous mode are, in some cases, very close to each other (e.g. the lower panels in figure 3), we do not exclude that the actual transition to turbulence could be porous-mode-induced. According to our results, an important clue of this shift should be the evidence of a larger set of excited wavenumbers. These issues go beyond the objectives of the present work.

In a future study, the present linear stability analysis should be completed by a suitable (weakly) nonlinear analysis in order to assess saturation mechanisms and explore the routes to turbulence. However, the theoretical study of transition to turbulence in porous-liquid interface systems by using the tools of hydrodynamic stability theory is just in its infancy, and these preliminary results have potential to find applications in several industrial (e.g. coating, heat exchange) and geophysical research (phase change systems) areas. We would like to mention just one particular case concerning glaciology.

As already argued in the seminal book by Hutter (1983), a free surface at which the ice is temperate involves a phase change system, which, in the case of melting, is composed of a layer of ice-water mixture (the porous medium) underneath the meltwater (the film). In this case the rate of melting of the surface is proportional to the jump of heat flux at the liquid-porous interface divided by $(1-\epsilon)$. Since both the heat flux and the porosity (or water content) are affected by viscous dissipation, it is straightforward to recognize that the discrimination between laminar or turbulent conditions in the porous layer is crucial for the rate of melting of the glacier surface. This particular problem requires a more involved thermo-fluid dynamic treatment than the present analysis and will be carried out in the future.

\section{Supplementary materials}

Supplementary materials are available at http://dx.doi.org/10.1017/jfm.2013.16. 


\section{REFERENCES}

AIDUn, C. K. 1991 Principles of hydrodynamic instability - application in coating systems. 2. Examples of flow instability. Tappi J. 74 (3), 213-220.

Beavers, G. S. \& Joseph, D. D. 1967 Boundary conditions at a naturally permeable wall. J. Fluid Mech. 30, 197-207.

Bender, C. M. \& Orszag, S. A. 1978 Advanced Mathematical Methods for Scientists and Engineers. McGraw-Hill.

Benjamin, J. 1957 Wave formation in laminar flow down an inclined plane. J. Fluid Mech. 2, $554-574$.

Camporeale, C., Canuto, C. \& Ridolfi, L. 2012 A spectral approach for the stability analysis of turbulent open-channel flows over granular beds. Theor. Comput. Fluid Dyn. 26, 51-80.

CAMporeale, C. \& Ridolfi, L. $2012 a$ Hydrodynamic-driven stability analysis of morphological patterns on stalactites and implications for cave paleoflow reconstructions. Phys. Rev. Lett. 108, 238501.

CAmporeale, C. \& Ridolfi, L. $2012 b$ Ice ripple formation at large Reynolds numbers. J. Fluid Mech. 27, 225-251.

Canuto, C., Hussaini, M. Y., Quarteroni, A. \& ZAng, T. A. 2006 Spectral Methods. Fundamentals in Single Domains. Springer.

D’ Alessio, S. J. D., Pascal, J. P., Jasmine, H. A. \& Ogden, K. A. 2010 Film flow over heated wavy inclined surfaces. J. Fluid. Mech. 665, 418-456.

Debruin, G. J. 1974 Stability of a layer of liquid flowing down an inclined plane. J. Engng Maths 8 (3), 259-270.

Devauchelle, O., Malverti, L., Lajeunesse, E., Lagree, P. Y., Josserand, C. \& ThU-LAM, K. D. N. 2010 Stability of bedforms in laminar flows with free surface: from bars to ripples. J. Fluid Mech. 642, 329-348.

Drazin, P. G. \& ReID, W. H. 1981 Hydrodynamic Stability. Cambridge University Press.

Floryan, J. M., Davis, S. H. \& Kelly, R. E. 1987 Instabilities of a liquid-film flowing down a slightly inclined plane. Phys. Fluids 30 (4), 983-989.

Giannakis, D., Fischer, P. F. \& Rosner, R. 2009 A spectral Galerkin method for the coupled Orr-Sommerfeld and induction equations for free-surface MHD. J. Comput. Phys. 228 (4), $1188-1233$.

Grosch, C. E. \& SALwen, H. 1968 The stability of steady and time-dependent plane Poiseuille flow. J. Fluid Mech. 34, 177-194.

HUtTER, K. 1983 Theoretical Glaciology: Material Science of Ice and the Mechanics of Glaciers and Ice Sheets. Springer.

Kelly, R. E., Goussis, D. A., Lin, S. P. \& HsU, F. K. 1989 The mechanism for surface-wave instability in film flow down an inclined plane. Phys. Fluids A: Fluid Dyn. 1 (5), 819-828.

LiU, R. \& LiU, Q. 2009 Instabilities of a liquid film flowing down an inclined porous plane. Phys. Rev. E 80 (3), 036316.

Manes, C., Poggi, D. \& Ridolfi, L. 2011 Turbulent boundary layers over permeable walls: scaling and near-wall structure. J. Fluid Mech. 687, 141-170.

Myers, T. G. 2003 Unsteady laminar flow over a rough surface. J. Engng Maths 46 (2), 111-126.

OCHOA-TAPiA, J. A. \& WhitAKer, S. 1995 Momentum-transfer at the boundary between a porous-medium and a homogeneous fluid. 1. Theoretical development. Intl J. Heat Mass Transfer 38 (14), 2635-2646.

Ogden, K. A., D’ Alessio, S. J. D. \& Pascal, J. P. 2011 Gravity-driven flow over heated, porous, wavy surfaces. Phys. Fluids 23 (12), 122102.

PASCAL, J. P. 1999 Linear stability of fluid flow down a porous inclined plane. J. Phys. D: Appl. Phys. 32 (4), 417-422.

Pokrajac, D. \& MANES, C. 2008 Interface between turbulent flows above and within rough porous walls. Acta Geophys. 56 (3), 824-844.

SAdiQ, I. M. R. \& Usha, R. 2008 Thin Newtonian film flow down a porous inclined plane: stability analysis. Phys. Fluids 20 (2), 022105.

Schmid, P. J. \& Henningson, D. S. 2001 Stability and transition in shear flows, Applied Mathematical Sciences, vol. 142. Springer. 
SHEN, J. 1994 Efficient spectral-Galerkin methods I. Direct solvers for the second and fourth order equations using Legendre polynomials. SIAM J. Sci. Comput. 15 (6), 1489.

SwarzTrauber, P. N. 2002 On computing the points and weights for Gauss-Legendre quadrature. SIAM J. Sci. Comput. 24 (3), 945-954.

Thiele, U., Goyeau, B. \& Velarde, M. G. 2009 Stability analysis of thin film flow along a heated porous wall. Phys. Fluids 21 (1), 014103.

Tilton, N. \& CORTElezzI, L. 2008 Linear stability analysis of pressure-driven flows in channels with porous walls. J. Fluid Mech. 604, 411-445.

Trevelyan, P. M. J., Scheid, B., Ruyer-Quil, C. \& Kalliadasis, S. 2007 Heated falling films. J. Fluid Mech. 592, 295-334.

Whitaker, S. 1986 Flow in porous media 1. A theoretical derivation of Darcy law. Trans. Porous Med. 1 (1), 3-25.

Whitaker, S. 1996 The Forchheimer equation: a theoretical development. Trans. Porous Med. 25 (1), 27-61.

YIH, S. 1955 Stability of two-dimensional parallel flows for three-dimensional disturbances. Q. Appl. Maths 12, 321.

YIH, S. 1963 Stability of liquid flow down an inclined plane. Phys. Fluids 6, 321. 